\title{
Cytokine Profiles Before and After Immune Modulation in Hospitalized Patients with COVID-19
}

\author{
Veronica Azmy ${ }^{1}$ (D) $\cdot$ Kelsey Kaman ${ }^{1} \cdot$ Daiwei Tang $^{2} \cdot$ Hongyu Zhao ${ }^{2} \cdot$ Charles Dela Cruz $^{3} \cdot$ Jeffrey E. Topal ${ }^{4,5}$. \\ Maricar Malinis ${ }^{4} \cdot$ Christina C. Price $^{1,6}$
}

Received: 3 October 2020 / Accepted: 16 December 2020 / Published online: 18 January 2021

(C) The Author(s), under exclusive licence to Springer Science+Business Media, LLC part of Springer Nature 2021

\begin{abstract}
We describe the cytokine profiles of a large cohort of hospitalized patients with moderate to critical COVID-19, focusing on IL-6, sIL2R, and IL-10 levels before and after receiving immune modulating therapies, namely, tocilizumab and glucocorticoids. We also discuss the possible roles of sIL2R and IL-10 as markers of ongoing immune dysregulation after IL-6 inhibition. We performed a retrospective chart review of adult patients admitted to a tertiary care center with moderate to critical SARS$\mathrm{CoV}-2$ infection. Disease severity was based on maximum oxygen requirement during hospital stay to maintain $\mathrm{SpO} 2>93 \%$ (moderate, 0-3 L NC; severe, 4-6 L NC or non-rebreather; critical, HFNC, NIPPV, or MV). All patients were treated using the institution's treatment algorithm, which included consideration of tocilizumab for severe and critical disease. The most common cytokine elevations among all patients included IL-6, sIL2R, IFN- $\gamma$, and IL-10; patients who received tocilizumab had higher incidence of IL-6 and sIL2R elevations. Pre-tocilizumab IL-6 levels increased with disease severity $(p=.0151)$. Both IL-6 and sIL2R levels significantly increased after administration of tocilizumab in all severity groups; IL-10 levels decreased in severe $(p=.0203)$, but not moderate or critical, patients after they received tocilizumab. Cluster analysis revealed association between higher admission IL-6, sIL2R, and CRP levels and disease severity. Mean IL-6, sIL2R, and D-dimer were associated with mortality, and tocilizumab-treated patients with elevated IL-6, IL-10, and D-dimer were more likely to also receive glucocorticoids. Accessible clinical cytokine panels may be useful for monitoring response to treatment in COVID-19. The increase in sIL2R post-tocilizumab, despite administration of glucocorticoids, may indicate the need for combination therapy in order to modulate more than one hyperinflammatory pathway in COVID-19. We also discuss the role of cytokines as potential biomarkers for use of adjunct glucocorticoid therapy.
\end{abstract}

Keywords COVID-19 - SARS-CoV-2 · cytokine release syndrome · interleukin-2 receptor (soluble) · interleukin-10 . tocilizumab $\cdot$ glucocorticoids $\cdot$ cytokine profile $\cdot$ cytokine panel

Veronica Azmy

veronica.azmy@yale.edu

1 Section of Rheumatology, Allergy \& Immunology, Yale University School of Medicine, TAC S469c, 333 Cedar Street, New Haven, CT 06511, USA

2 Department of Biostatistics, Yale School of Public Health, Yale University, New Haven, CT, USA

3 Section of Pulmonary, Critical Care and Sleep Medicine, Yale University School of Medicine, New Haven, CT, USA

4 Section of Infectious Diseases, Yale University School of Medicine, New Haven, CT, USA

5 Department of Pharmacy Services, Yale New Haven Hospital, New Haven, CT, USA

6 Department of Allergy and Immunology, VA Medical Center, West Haven, CT, USA

\section{Introduction}

Severe acute respiratory syndrome coronavirus 2 (SARS$\mathrm{CoV}-2)$ is the novel coronavirus responsible for the coronavirus disease 2019 (COVID-19) global pandemic, which has claimed over 120,000 lives in the USA as of June 26, 2020 [1]. Studies looking at predictors of mortality have suggested that a subset of patients with COVID-19 may suffer from systemic hyperinflammation, akin to a cytokine release syndrome (CRS) seen in chimeric antigen receptor (CAR) T cell therapy [2] or the cytokine storm seen in macrophage activation syndrome (MAS) or secondary hemophagocytic lymphohistiocytosis (sHLH) [3]. This systemic hyperinflammation is important in the pathogenesis of acute respiratory distress syndrome (ARDS), the leading cause of 
mortality in COVID-19 [4]. These syndromes involve elevations in several cytokines, many of which have also been implicated in COVID-19 inflammation.

CRS is a systemic immune response that can be triggered by various factors and is a proposed mechanism for the pathophysiology of severe viral infections such as influenza $[5,6]$. In patients with CRS, interleukin (IL)-6, IL-10, and interferon (IFN)- $\gamma$ are consistently elevated [2]. In particular, IL-6, a proinflammatory cytokine with a pleiotropic effect on the immune system, contributes to many of the symptoms observed, such as the production of acute phase reactants by hepatocytes [7], activation of the extrinsic coagulation pathway [8], and production of vascular endothelial growth factor (VEGF), leading to angiogenesis and endothelial inflammation [9]. Based on several recent studies, IL-6 has been implicated as a predictive marker of morbidity and mortality in SARS-CoV2 infection [10,11], and for this reason, tocilizumab, a humanized monoclonal antibody against the IL- 6 receptor, is being investigated as possible treatment for COVID-19 pneumonia [12]. However, other cytokines which are not targeted by tocilizumab, such as IL-2-, IL-10-, and IFN- $\gamma$-induced protein 10 (IP-10), have also been associated with more critical disease [13].

IL-10 has several key functions in the immune response. In the setting of infection, it suppresses macrophage and dendritic cell activation and limits Th1 and Th2 effector responses. It does so by curbing two major cytokine effector pathways, $\mathrm{NF}-\kappa \mathrm{B}$ and JAK-STAT, thereby counteracting the hyperactive immune response $[14,15]$. This results in protection for the host by limiting tissue damage caused by ongoing inflammation but also promotes persistence of infection. There is additional evidence that the intensity of the IL-10 response reflects the strength of the preceding inflammation [14]. In COVID-19, IL-10 is likely upregulated in the setting of overwhelming inflammation but may also contribute to the development of lung fibrosis and the infiltration of inflammatory cells into the lungs [16]. Furthermore, little is known regarding the timing of IL-10 secretion in COVID-19, which may be important given that early production may lead to overwhelming infection and ongoing production after viral containment may result in pathology [14].

Although cytokines have been associated with several disease processes, little is known regarding their use in monitoring and assessing response to therapy in the clinical setting. Given the complex ways in which they interact within the immune system, it is often difficult to assess their proinflammatory versus anti-inflammatory functions and generate appropriate cutoffs for normal values [17]. There are a number of immunoassay and bioassay methods used to detect cytokine levels, the most common of which are enzymelinked immunosorbent assays (ELISA), multiplex arrays, bead-based assays, and immunosensing methods. To date, cytokines have been used in the diagnosis and management of a variety of infections including tuberculosis, ventilatorassociated pneumonia, and neonatal sepsis, as well as chronic diseases such as Alzheimer's disease, gastric cancer, and HLH, to name a few [17]. Given what we already know about cytokine levels in CRS and our relatively limited understanding of SARS-CoV-2, the cytokine profiles of hospitalized patients with COVID-19 may prove useful for guiding management. In this paper, we describe the cytokine profiles of a previously reported large cohort of hospitalized patients with moderate to critical COVID-19 [18], particularly focusing on their IL-6, sIL2R, and IL-10 levels before and after receiving immune modulating therapy. We also discuss the possible role of IL-10 as a marker of ongoing immune dysregulation after IL-6 inhibition.

\section{Methods}

\section{Study Design and Participants}

Adults 18 years of age or above with PCR-confirmed SARS$\mathrm{CoV}-2$ infection consecutively admitted to a tertiary care hospital between March 10 and 31, 2020, in New Haven, CT, USA, underwent standardized chart review. Yale School of Medicine Institutional Review Board (2000027792) approved this study. Patients were identified through the electronic medical record (EMR) and underwent 21-day observation period with follow-up data through April 21, 2020. All patients had been treated under the institution's treatment algorithm, which was formed by a multidisciplinary team based on the available literature during the study period [18]. The algorithm included consideration for using tocilizumab, an IL-6 antagonist, in treatment of COVID-19 in patients who required supplemental oxygen $\mathrm{SpO} 2 \geq 3 \mathrm{~L}$ to maintain $\mathrm{SpO} 2>93 \%$. Tocilizumab was chosen based on biological plausibility, potential utility in treating CRS associated with COVID-19, and a favorable safety profile. Based on the treatment algorithm, tocilizumab was administered $8 \mathrm{mg} / \mathrm{kg}$ intravenously, not to exceed $800 \mathrm{mg}$, with an optional second dose for patients with body mass index (BMI) above $40 \mathrm{~kg} / \mathrm{m}^{2}$.

Cytokine panels were collected as part of this institution's COVID-19 treatment algorithm, which specified collection upon admission and then every $48 \mathrm{~h}$ thereafter. This was done to assess for cytokine release syndrome and to monitor progression throughout hospitalization. The panel included interleukin-2 receptor (CD25) soluble (sIL2R), IL-12, IFN- $\gamma$, IL-4, IL-5, IL-10, IL-13, IL-1 beta, IL-6, IL-8, tumor necrosis factor (TNF)-alpha, IL-2, and IL-17. Cytokine panel tests were performed via a quantitative multiplex bead assay by a CLIA-certified diagnostic lab in Salt Lake City, UT. Lower limit of detection was $5 \mathrm{pg} / \mathrm{mL}$. 


\section{Data Collection}

We performed a structured chart review which included demographic data, health history, date of medication administration, oxygen requirements, and serial cytokine levels.

\section{Definitions}

Moderate illness was defined as room air up to maximum requirement of $3 \mathrm{~L} / \mathrm{min}$ oxygen via nasal cannula (NC) to maintain $\mathrm{SpO} 2>93 \%$. Severe illness was defined as requirement of 4 to $6 \mathrm{~L} / \mathrm{min}$ or a non-rebreather $(100 \% \mathrm{SpO} 2)$ to maintain $\mathrm{SpO} 2>93 \%$, and critical illness was defined as requiring high-flow nasal cannula (HFNC), non-invasive positive pressure ventilation (NIPPV) or mechanical ventilation (MV). HFNC and NIPPV were included in the same severity category as MV given that the majority of patients placed on these therapies progressed to NIPPV unless their goals of care did not align. The highest level of support required during hospitalization was used to group patient severity. Elevated IL-6, IL-1 beta, IL-12, IL-13, IL-17, IL-2, IL-4, IL-5, and IL-8 levels were defined as greater than $5 \mathrm{pg} / \mathrm{mL}$. Elevated IL-10 was defined as greater than $18 \mathrm{pg} / \mathrm{mL}$, and elevated sIL2R was defined as greater than $1033 \mathrm{pg} / \mathrm{mL}$. A patient was defined to have elevation of a specific cytokine if the average level of that cytokine throughout their hospital admission was elevated. Pre- and post-tocilizumab values were obtained by taking the average of all levels before and after patients received treatment, respectively. A patient was defined to have received glucocorticoids if they received a steroid dose equivalent to $\geq 20 \mathrm{mg} /$ day of oral prednisone.

\section{Statistical Analysis}

We hypothesized that hospitalized patients with COVID-19 would have elevations in IL-6, sIL2R, and IL-10 and that levels would differ by disease severity. Our secondary hypothesis was that IL-6 and IL-10 levels would change pre- and post-tocilizumab, but that sIL2R levels would be less affected. For comparison across severity groups, we performed oneway ANOVA for continuous variables and chi-square to compare categorical data. We calculated the number of patients in each severity group who had elevation in each cytokine. For cytokine level comparisons pre- and post-tocilizumab, we used Welch two-group $t$ test assuming unequal variances. Given the time series nature of our data, each cytokine level was used as an individual data point for the purpose of testing our hypothesis. For clustering data pre-processing, we first chose five cytokines (IL-6, IL-10, sIL2R, CRP, and d-dimer) which did not have substantial missing or undetectable values as our features, and we did not include patients with missing values for any of the cytokines. For each patient, we performed cluster analysis via two methods: first, using each initial cytokine value on admission, and second, the mean cytokine value throughout admission. Given the huge scale difference across cytokines, we normalized each cytokine level to a range between -5 and 5 . All analyses were performed using $\mathrm{R}$ version 3.6.1.

\section{Results}

The cytokine profiles of 239 consecutive hospitalized patients with COVID-19 were collected. Basic demographic and health data can be found in Table 1 . There were 114 patients identified as having moderate disease, 54 with severe disease, and 71 with critical disease. The median age of this cohort was 64 years (range 22-99 years). The moderate group was younger than the severe and critical groups, with a median age of 60 compared with 67.5 and 64.5 , respectively $(p=.002)$. Among the critical group, there was a male predominance, with $62 \%$ males, although this was not statistically significant; in the moderate and severe groups, the sex distribution was more equal. The racial distribution of the overall population was $37 \%$ black, $46 \%$ white, $2 \%$ Asian or Pacific Islander, and $4 \%$ other. Eleven percent of patients identified as Hispanic. There were no statistically significant differences in race between severity groups. Among all patients, $65 \%$ were obese, $59 \%$ had hypertension, 38\% had diabetes, 38\% had chronic lung disease, and $30 \%$ had chronic heart disease. BMI was higher in the critical group compared with the moderate and severe groups (33 versus 29 and 30, respectively; $p=.007$ ). There were no other statistically significant differences between groups with regard to medical comorbidities. Of all patients, $64 \%$ received tocilizumab, $34 \%$ of moderate patients, $87 \%$ of severe patients, and $93 \%$ of critical patients $(p<.00001)$. Thirty-five percent of all patients treated with tocilizumab also received glucocorticoids (16\% moderate, $36 \%$ severe, $47 \%$ critical). Fourteen-day survival among all tocilizumab-treated patients was $88 \%$ (100\% moderate, $91 \%$ severe, $82 \%$ critical).

Table 2 displays the proportion of patients with elevations in each cytokine, further grouped by disease severity and whether or not they received tocilizumab. Among patients with moderate disease, 39 (34\%) received tocilizumab while $75(66 \%)$ did not. In the severe group, 48 patients $(89 \%)$ received tocilizumab while 6 patients $(11 \%)$ did not. Finally, in the critical group, 66 patients $(93 \%)$ received tocilizumab while 5 patients (7\%) did not. Among all patients, $77 \%$ had elevated IL-6 during their hospital stay $(64.9 \%$ of moderate patients, $83.3 \%$ of severe patients, and $91.5 \%$ of critical patients), making IL-6 the most commonly seen cytokine elevation among these hospitalized patients. In all three severity groups, those who received tocilizumab had higher proportions of patients with IL- 6 elevation than those who did not receive tocilizumab (79.5\% moderate, $81.2 \%$ severe, $90.9 \%$ 
Table 1 Characteristics of COVID-19 infected patients

\begin{tabular}{|c|c|c|c|c|c|}
\hline & $\begin{array}{l}\text { All } \\
(N=239)\end{array}$ & $\begin{array}{l}\text { Moderate* } \\
(N=114)\end{array}$ & $\begin{array}{l}\text { Severe** } \\
(N=54)\end{array}$ & $\begin{array}{l}\text { Critical*** } \\
(N=71)\end{array}$ & $p$ value \\
\hline \multicolumn{6}{|l|}{ Patient characteristics } \\
\hline Age, median (range), years & $64(22-99)$ & $60(23-99)$ & $67.5(23-92)$ & $64.5(22-93)$ & .002 \\
\hline Gender, No. (\%) & & & & & .154 \\
\hline Female & $113(43)$ & $60(53)$ & $26(48)$ & $27(38)$ & \\
\hline Male & $126(53)$ & $54(47)$ & $28(52)$ & $44(62)$ & \\
\hline Race/ethnicity, No. (\%) & & & & & .287 \\
\hline Black & $88(37)$ & $42(37)$ & $15(28)$ & $31(44)$ & \\
\hline White & $110(46)$ & $5(44)$ & $31(57)$ & $29(41)$ & \\
\hline Asian/Pacific Islander & $4(2)$ & $3(3)$ & $0(0)$ & $1(1)$ & \\
\hline Other & $10(4)$ & $5(4)$ & $4(7)$ & $1(1)$ & \\
\hline Hispanic & $27(11)$ & $14(12)$ & $4(7)$ & $9(13)$ & \\
\hline \multicolumn{6}{|l|}{ Medical comorbidities } \\
\hline Diabetes, No. (\%) & $91(38)$ & $36(32)$ & $22(41)$ & $33(46)$ & .116 \\
\hline $\begin{array}{l}\text { Uncontrolled diabetes defined by } \mathrm{hgA} 1 \mathrm{c} \geq 8 \mathrm{mg} / \mathrm{dL} \text {, No. } \\
(\%)\end{array}$ & $40(17)$ & $13(11)$ & $11(20)$ & $16(23)$ & .100 \\
\hline Immunosuppressed, No. (\%) & $36(15)$ & $15(13)$ & $6(11)$ & $15(21)$ & .2223 \\
\hline Chronic lung disease, No. (\%) & $91(38)$ & $41(36)$ & $26(48)$ & $24(34)$ & .215 \\
\hline Hypertension, No. (\%) & $142(59)$ & $64(56)$ & $32(59)$ & $46(65)$ & .589 \\
\hline Chronic heart disease, No. (\%) & $71(30)$ & $39(34)$ & $11(20)$ & $21(30)$ & .188 \\
\hline Obesity (BMI $\geq 30)$, No. $(\%)$ & $156(65)$ & $68(60)$ & $35(65)$ & $53(75)$ & .110 \\
\hline Body mass index, mean (SD) & $31( \pm 7.3)$ & $29( \pm 6.3)$ & $30( \pm 7.9)$ & $33( \pm 7.6)$ & .007 \\
\hline BMI classification, No. (\%) & & & & & .280 \\
\hline$<30$ & $75(32)$ & $40(37)$ & $19(35)$ & $16(23)$ & \\
\hline $30.0-34.99$ & $101(42)$ & $45(42)$ & $23(43)$ & $33(48)$ & \\
\hline $35.0-39.99$ & $33(14)$ & $16(15)$ & $8(15)$ & $9(13)$ & \\
\hline 40 or more & $22(9)$ & $7(6)$ & $4(7)$ & $11(16)$ & \\
\hline Tocilizumab, No. (\%) & $153(64)$ & $39(34)$ & $47(87)$ & $66(93)$ & - \\
\hline Glucocorticoids among tocilizumab-treated, No. (\%) & $54(35)$ & $6(16)$ & $17(36)$ & $31(47)$ & - \\
\hline Survival (14-day) among tocilizumab-treated (CI 95\%) & $134(88)$ & $37(100)$ & $43(91)$ & $54(82)$ & - \\
\hline Survival (14-day) among all patients & $214(90)$ & $112(98)$ & $46(85)$ & $56(79)$ & - \\
\hline
\end{tabular}

Tests used: chi-square for categorical data, one-way ANOVA for continuous variables

* Moderate disease defined as room air up to $3 \mathrm{~L} \mathrm{NC}$ at any point during hospital stay

**Severe disease defined as 4 to $6 \mathrm{~L} \mathrm{NC}$ or non-rebreather at any point during hospital stay

***Critical disease defined as requiring HFNC, NIPPV, or MV at any point during hospital stay

severe). With regard to sIL2R, it was elevated in $50.6 \%$ of all patients (37.7\% moderate, $53.7 \%$. severe, and $69.0 \%$ critical). IL-10 was elevated in $22.6 \%$ of all patients ( $11.4 \%$ moderate, $24.1 \%$ severe, and $39.4 \%$ critical). IFN- $\gamma$ was elevated in $29.3 \%$ of all patients, and IL-1 beta was elevated in $16.7 \%$. IL-2 was elevated in $23.0 \%$ of all patients at a similar proportion throughout all severity groups. TNF-alpha and IL-12 were elevated in $14.2 \%$ and $9.6 \%$ of all patients, respectively. IL-13, IL-4, IL-5, and IL-8 were less commonly seen overall (4.2\%, $4.6 \%, 4.2 \%$, and $6.7 \%$, respectively).

Figure 1 displays mean cytokine concentrations pre- and post-tocilizumab grouped by disease severity, with further elaborations in Table 3. With regard to IL-6 (Fig. 1a), pretocilizumab levels increased with disease severity $(19.5 \mathrm{pg} /$ $\mathrm{mL}$ moderate, $21.2 \mathrm{pg} / \mathrm{mL}$ severe, and $42.4 \mathrm{pg} / \mathrm{mL}$ critical; $p=.015)$ After administration of tocilizumab, IL-6 levels rose in all three groups compared with pre-tocilizumab levels (moderate, $120.68 \mathrm{pg} / \mathrm{mL}, p=.009$; severe, $163.97 \mathrm{pg} / \mathrm{mL}$, $p<.001$; critical, $212.5 \mathrm{pg} / \mathrm{mL}, p<.001)$. Pre-tocilizumab sIL2R levels (Fig. 1b) increased with disease severity with a trend toward significance (moderate, $1027.56 \mathrm{pg} / \mathrm{mL}$; severe, $1136.92 \mathrm{pg} / \mathrm{mL}$; critical, $1315.68 \mathrm{pg} / \mathrm{mL}, p=.153)$, and levels also rose post-tocilizumab (moderate, $1525 \mathrm{pg} / \mathrm{mL}, p=.011$; severe, $1402.68 \mathrm{pg} / \mathrm{mL}, p=.046$; critical, $1776.24 \mathrm{pg} / \mathrm{mL}$, 
Table 2 Number of COVID-19 patients with individual cytokine elevation

\begin{tabular}{|c|c|c|c|c|c|c|c|}
\hline \multirow[b]{2}{*}{ Cytokine } & \multirow{2}{*}{$\begin{array}{l}\text { All } \\
(N=239)\end{array}$} & \multicolumn{2}{|c|}{ Moderate $(N=114)$} & \multicolumn{2}{|c|}{ Severe $(N=54)$} & \multicolumn{2}{|c|}{ Critical $(N=71)$} \\
\hline & & $\begin{array}{l}\text { Toci } \\
(N=39)\end{array}$ & $\begin{array}{l}\text { No Toci } \\
(N=75)\end{array}$ & $\begin{array}{l}\text { Toci } \\
(N=48)\end{array}$ & $\begin{array}{l}\text { No Toci } \\
(N=6)\end{array}$ & $\begin{array}{l}\text { Toci } \\
(N=66)\end{array}$ & $\begin{array}{l}\text { No Toci } \\
(N=5)\end{array}$ \\
\hline IL-6, No. (\%) & $184(77.0)$ & $34(87.2)$ & $40(53.3)$ & $41(85.4)$ & $4(66.7)$ & $61(92.4)$ & $4(80.0)$ \\
\hline sIL2R, No. (\%) & $121(50.6)$ & $17(43.6)$ & $26(34.6)$ & $26(54.2)$ & $3(50.0)$ & $48(72.7)$ & $1(20.0)$ \\
\hline IL-10, No. (\%) & $54(22.6)$ & $4(10.3)$ & $9(12.0)$ & $13(27.1)$ & $0(0.0)$ & $25(37.9)$ & $3(60.0)$ \\
\hline IFN-Y, No. (\%) & $70(29.3)$ & $11(28.2)$ & $14(18.7)$ & $18(37.5)$ & $0(0.0)$ & $27(40.9)$ & $0(0.0)$ \\
\hline IL-1 B, No. (\%) & $41(17.2)$ & $5(12.8)$ & $13(17.3)$ & $9(18.8)$ & $0(0.0)$ & $13(19.7)$ & $1(20.0)$ \\
\hline IL-12, No. (\%) & $23(9.6)$ & $3(7.7)$ & $7(9.3)$ & $7(14.6)$ & $0(0.0)$ & $6(9.1)$ & $0(0.0)$ \\
\hline IL-13, No. (\%) & $10(4.2)$ & $1(2.6)$ & $2(2.7)$ & $2(4.1)$ & $0(0.0)$ & $5(7.6)$ & $0(0.0)$ \\
\hline IL-17, No (\%) & $34(14.2)$ & $3(7.7)$ & $14(18.7)$ & $5(10.4)$ & $0(0.0)$ & $10(15.2)$ & $2(40.0)$ \\
\hline IL-2, No (\%) & $60(25.1)$ & $11(28.2)$ & $15(20.0)$ & $14(29.2)$ & $0(0.0)$ & $19(28.8)$ & $1(20.0)$ \\
\hline IL-4, No. (\%) & $12(5.0)$ & $1(2.5)$ & $3(4.0)$ & $4(8.3)$ & $0(0.0)$ & $4(6.1)$ & $0(0.0)$ \\
\hline IL-5, No. (\%) & $11(4.6)$ & $1(2.5)$ & $2(2.7)$ & $2(4.2)$ & $0(0.0)$ & $6(9.1)$ & $0(0.0)$ \\
\hline IL-8, No. (\%) & $17(7.1)$ & $3(7.7)$ & $1(1.3)$ & $2(4.2)$ & $0(0.0)$ & $11(16.7)$ & $0(0.0)$ \\
\hline TNF-a, No. (\%) & 35 (14.6) & $2(5.1)$ & $11(14.7)$ & $10(20.8)$ & $0(0.0)$ & $11(16.7)$ & $1(20.0)$ \\
\hline
\end{tabular}

$p=.0019$ ). With regard to IL-10 (Fig. 1c), pre-tocilizumab IL10 concentrations were $14 \mathrm{pg} / \mathrm{mL}$ in the moderate group, $31.94 \mathrm{pg} / \mathrm{mL}$ severe, and $20.25 \mathrm{pg} / \mathrm{mL}$ critical $(p=.1)$. After tocilizumab, the concentrations decreased in all three groups (12.25 pg/mL moderate, $10.87 \mathrm{pg} / \mathrm{mL}$ severe, $15.9 \mathrm{pg} / \mathrm{mL}$ critical), but the change was found to be statistically significant only in the severe group $(p=.0203)$. Ferritin levels trended up with disease severity, but were not statistically significant (Table 3) (moderate, 1033 pg/mL; severe, 1204 pg/mL; critical, 1603 pg/mL, $p=.0673$ ). Ferritin levels also trended toward an increase in all severity groups after tocilizumab, but results were not significant.

Mean IL-6 concentration over time in patients who received tocilizumab is displayed in Fig. 2a. Maximum IL-6 levels were higher in critical patients compared with moderate and severe patients, as evidenced by the higher peak levels. Additionally, IL-6 levels generally began to rise few days after symptom onset, as portrayed graphically in the figure. In severe and critical patients, IL- 6 remained elevated 20 days after symptom onset but were lower than earlier in the disease course. Mean IL-10 concentration over time in those who received tocilizumab is shown in Fig. 2b Peak IL-10 levels occurred earlier in disease course and gradually decreased over time. Additionally, in severe and critical patients, they remained elevated even after 20 days from symptom onset.

Results from our cluster analysis are presented in Fig. 3. Hierarchic clustering using Euclidean distance did not give any meaningful signals. When rearranged by severity, high admission IL-6, sIL2R, and CRP levels clustered into the severe and critical groups (Fig. 3a). When looking at mortality
Table 3 Cytokine concentrations pre- and post-tocilizumab by disease severity

\begin{tabular}{llllll}
\hline & & Moderate $(N=39)$ & Severe $(N=48)$ & Critical $(N=66)$ & $p$ value \\
\hline IL-6 $(\mathrm{pg} / \mathrm{mL})$ & Pre & 19.5 & 21.2 & 42.4 & .0151 \\
& Post & 120.68 & 163.97 & 212.5 & .0334 \\
& $p$ value & .009 & $<.001$ & $<.001$ & \\
SIL2R $(\mathrm{pg} / \mathrm{mL})$ & Pre & 1027.56 & 1136.92 & 1315.68 & .153 \\
& Post & 1525.2 & 1402.68 & 1776.24 & .0392 \\
& $p$ value & .011 & .046 & .0019 & .1 \\
IL-10 $(\mathrm{pg} / \mathrm{mL})$ & Pre & 14 & 31.94 & 20.25 & .179 \\
& Post & 12.25 & 10.87 & 15.9 & .2136 \\
& $p$ value & .7604 & .0203 & 1603 & 0.0673 \\
Ferritin $(\mathrm{pg} / \mathrm{mL})$ & Pre & 1033 & 1204 & 3828 & 0.136 \\
& Post & 1492 & 1685 & 0.1539 & \\
\hline
\end{tabular}



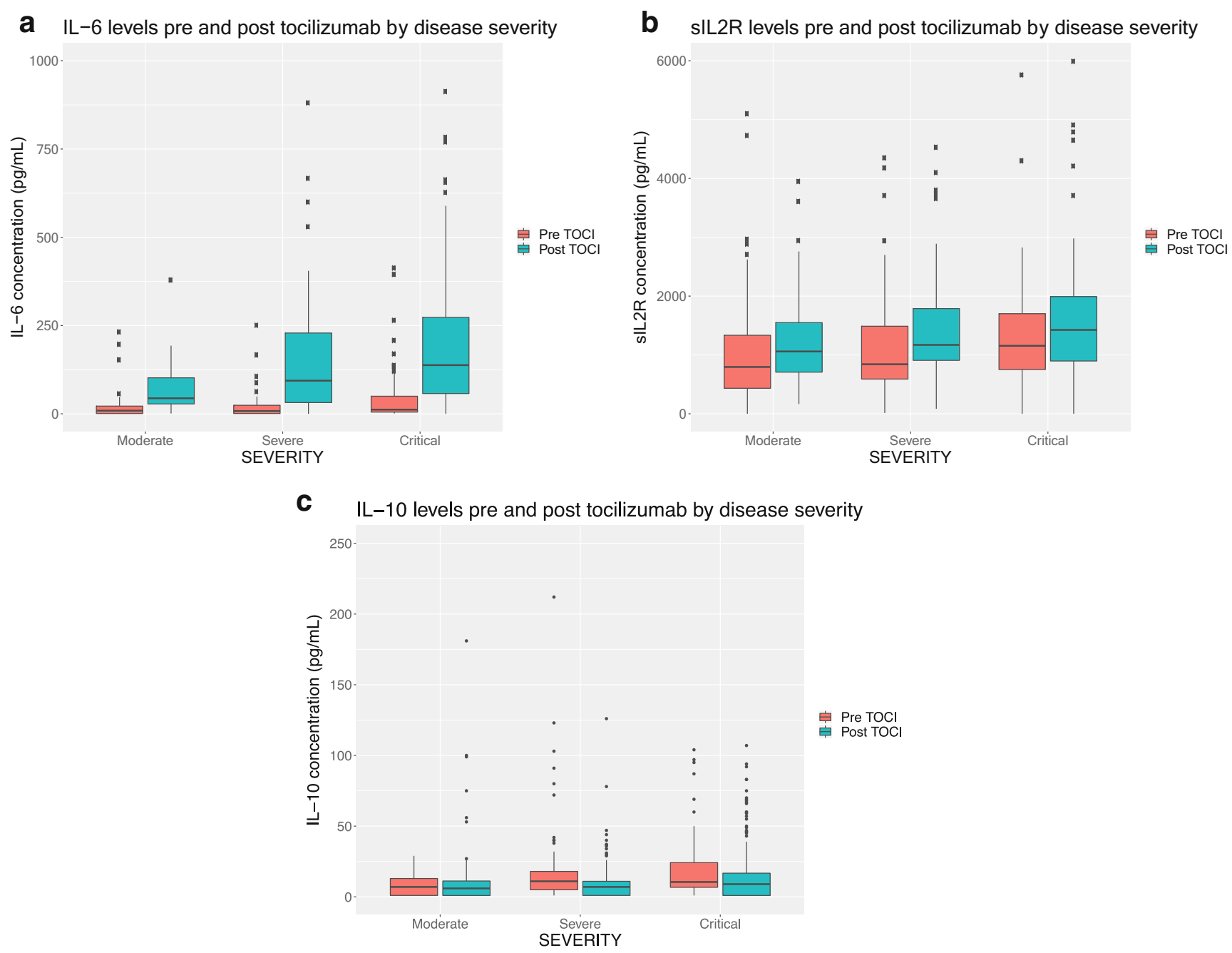

Fig. 1 Cytokine levels pre and post tocilizumab by disease severity. a IL6: Pre-tocilizumab levels increased with disease severity $(19.5 \mathrm{pg} / \mathrm{mL}$ moderate, $21.2 \mathrm{pg} / \mathrm{mL}$ severe, and $42.4 \mathrm{pg} / \mathrm{mL}$ critical; $p=.015$ ). After administration of tocilizumab, IL-6 levels rose in all three groups (moderate, $120.68 \mathrm{pg} / \mathrm{mL}, p=.009$; severe, $163.97 \mathrm{pg} / \mathrm{mL}, p<.001$; critical, $212.5 \mathrm{pg} / \mathrm{mL}, p<.001)$. b sIL2R: Pre-tocilizumab sIL2R levels increased with disease severity with a trend toward significance (moderate, $1027.56 \mathrm{pg} / \mathrm{mL}$; severe, $1136.92 \mathrm{pg} / \mathrm{mL}$; critical, $1315.68 \mathrm{pg} / \mathrm{mL}$,

$p=.153$ ). Levels also rose post-tocilizumab in all three groups (moderate, $1525 \mathrm{pg} / \mathrm{mL}, p=.011$; severe, $1402.68 \mathrm{pg} / \mathrm{mL}, p=.046$; critical, $1776.24 \mathrm{pg} / \mathrm{mL}, p=.0019)$. c IL-10: There were no differences in pretocilizumab IL-10 levels between groups. After tocilizumab, the concentrations decreased in all three groups $(-12.25 \mathrm{pg} / \mathrm{mL}$ moderate, $10.87 \mathrm{pg} / \mathrm{mL}$ severe, $-15.9 \mathrm{pg} / \mathrm{mL}$ critical), but the change was found to be statistically significant only in the severe group $(p=.0203)$

as outcome, there were no apparent signals with initial cytokine levels (Fig. 3b), but when using mean cytokine levels, higher mean IL-6, sIL2R, IL-10, and D-dimer clustered with the death outcome (Fig. 3c). Finally, among those who received tocilizumab, patients who also received glucocorticoids had higher initial D-dimer, IL-6, and IL-10 levels (Fig. 3d).

\section{Discussion}

This retrospective study describes the cytokine profiles of 239 hospitalized patients with moderate to critical COVID-19 and the changes in IL-6, sIL2R, and IL-10 before and after immune modulation in 153 patients. The core cytokines found to be elevated using our clinical-based cytokine assay were IL-6, sIL2R, IFN- $\lambda$, and IL-10, with others, such as IL-1 $\beta$, IL-2, and IL-17, in a much smaller subset of patients. A recent paper which identified a "core COVID-19 signature" among moderate and severe patients corroborates our findings with the addition of several other cytokines, IL- $1 \alpha$, IL-12 p70, IFN $\alpha$, IL-18, and TNF [19]. The reason for the difference may lie in the fact that their cytokines were obtained using an in-depth research-based assay, while we obtained our results using a certified clinical assay, accessible to the average clinician, which was our aim.

Although we reported differential profiles for patients who did and did not receive tocilizumab, given this was a 
Fig. 2 Cytokine trends in patients who received tocilizumab. a Mean IL-6 over time by severity in patients who received tocilizumab. Maximum IL-6 levels were higher in critical patients compared with moderate and severe patients. IL-6 levels began to rise few days after symptom onset. b Mean IL-10 over time by severity in patients who received tocilizumab. Peak IL-10 levels occurred earlier in disease course and gradually decreased over time

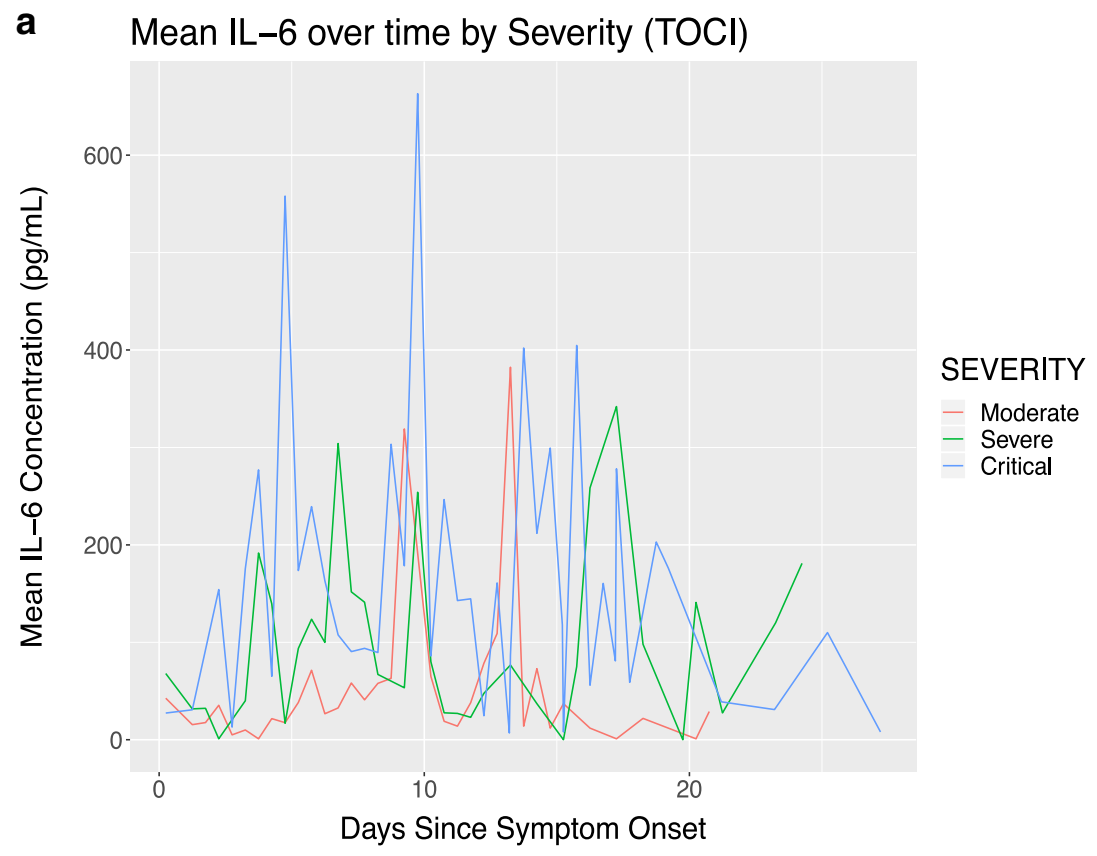

b Mean IL-10 over time by Severity (TOCI)

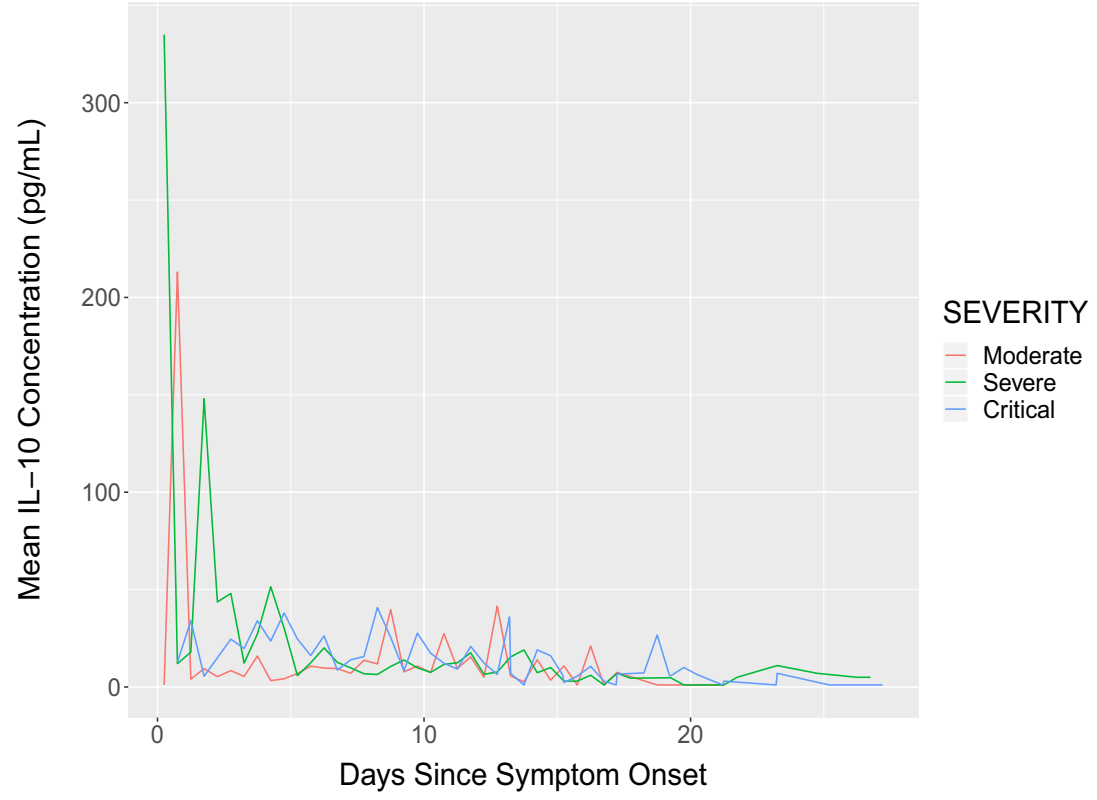

retrospective study and not a randomized controlled trial, a comparison between these groups are somewhat limited. Among all patients who received tocilizumab, 14-day survival was $88 \%$. A higher proportion of these patients had elevated IL-6 and sIL2R, which likely reflects the inherently more severe nature of their disease. With regard to IL-10, a higher proportion of patients with critical disease who did not receive tocilizumab had elevation, indicating that tocilizumab did in fact calm the cytokine storm and need for anti-inflammatory action. IFN- $\gamma$ and IL-1 beta were also elevated in a higher proportion of patients who received tocilizumab, so it seems that these patients who were selected to receive it based on oxygen requirement did in fact also have a more severe CRS, supporting the idea that CRS is a major factor in the development of ARDS.

Moreover, pre-tocilizumab IL-6 levels increased with disease severity, confirming findings of earlier studies, which found IL-6 to be a predictive marker of severity in COVID19 patients $[10,11]$. The increased IL-6 levels after administration of tocilizumab are expected. Prior studies in 

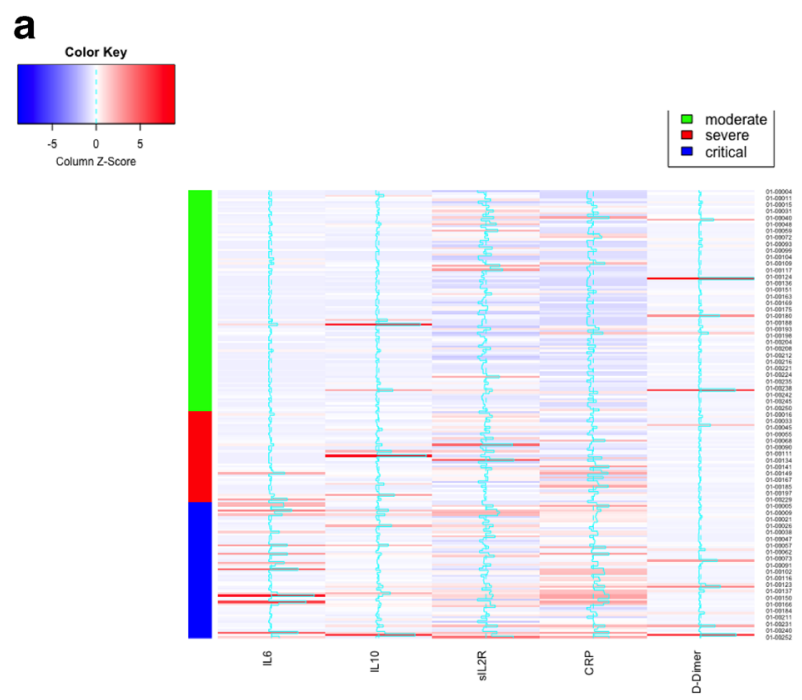

b
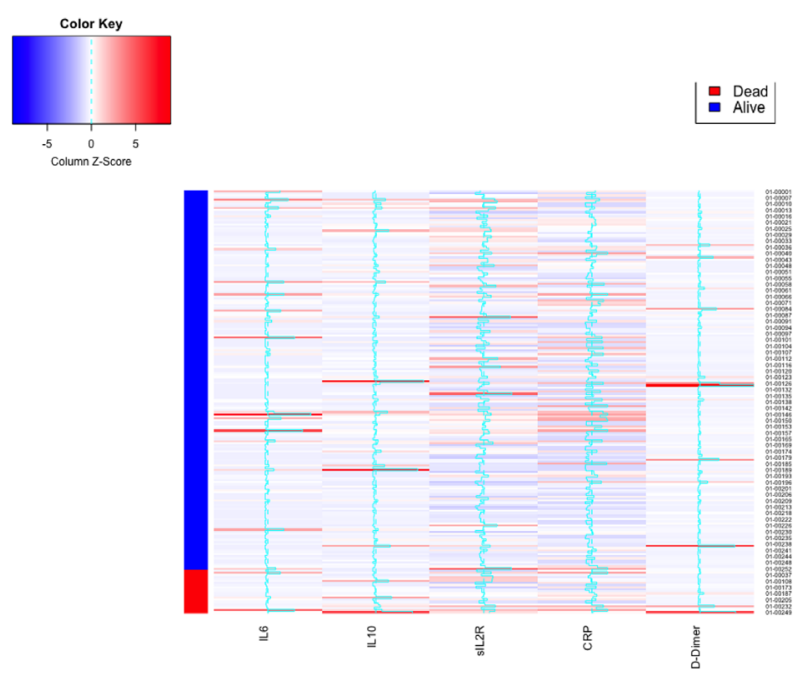

Fig. 3 Cluster analysis for severity, mortality, and glucocorticoid status. a Disease severity and initial cytokine level. Higher admission IL-6, sIL2R, and CRP levels clustered into the severe and critical disease groups. b Mortality and initial cytokine level. When looking at mortality as outcome, there were no apparent signals with initial cytokine levels. c

rheumatoid arthritis and Castleman disease found serum IL-6 increased after tocilizumab administration because of a decrease in tocilizumab-free IL-6 receptors, which would normally bind IL-6 and lead to its consumption [20].

On the other hand, the reason behind the increase in SIL2R after tocilizumab is less clear. Very high sIL2R, a marker for T cell activation and proliferation, is rarely seen outside the context of HLH [21], so this observation makes these high levels seen in COVID-19 hyperinflammation worth further exploration. Among those with elevated sIL2R, a subset did have hyperferritinemia, but we did not see an increase in ferritin levels with disease severity, making it unlikely HLH-related. Other markers of HLH, including triglycerides, fibrinogen,
C

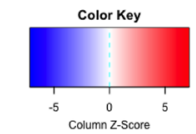

$\mid$ E. Dead

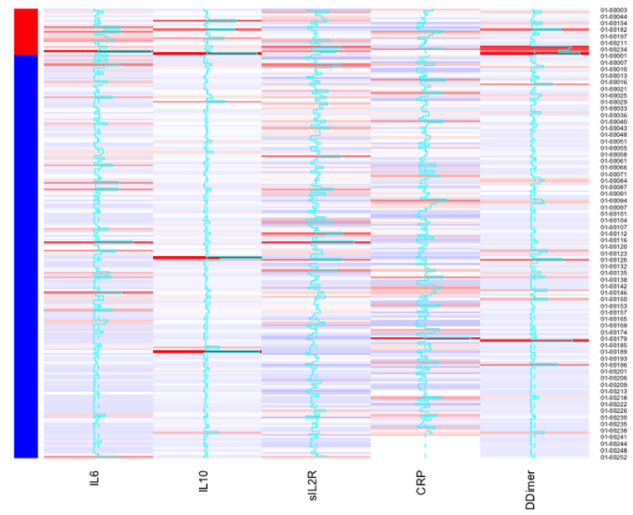

d

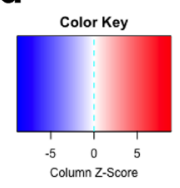

- Non-glucocorticoids

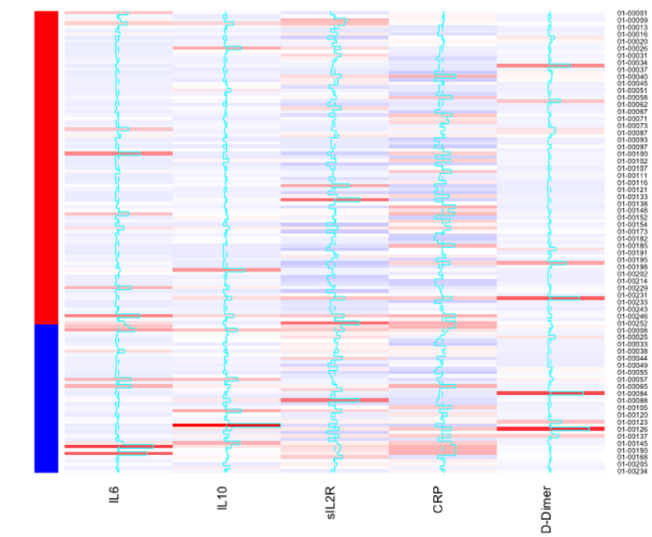

Mortality and mean cytokine level. Higher mean IL-6, sIL2R, IL-10, and D-dimer clustered with the death outcome. d Tocilizumab-treated patients, glucocorticoid status, and initial cytokine level. Patients who also received glucocorticoids had higher initial D-dimer, IL-6, and IL-10 levels

and splenomegaly, were measured in the first few patients we observed very elevated sIL2R levels, but given that they did not meet HLH criteria, we did not continue obtaining these markers on subsequent patients. The cause of the sIL2R rise needs to be further investigated as treatment for severe and critical disease may need to target this pathway in addition to the IL-6 pathway.

Our study found that IL-10 decreased after tocilizumab administration in severe, but not moderate or critical, patients with SARS-CoV-2 infection. With regard to the moderate group, a change was likely not seen due to the small number of patients in that cohort who received tocilizumab. Perhaps with larger sample size, we would have seen a decrease in IL- 
10 in the moderate group. There are several possible explanations for why the critical patients did not have a decrease in IL10 after tocilizumab. First, timing of tocilizumab administration may have played a role. Prior prospective studies have found that tocilizumab was less effective in critical patients and that use of tocilizumab earlier in the disease course proved more beneficial $[22,23]$. More likely, it is possible that in these critical patients, tocilizumab alone was inadequate in calming the inflammation caused by the disease, as evidenced by elevations in cytokines from other immune pathways. Another possible explanation is that critical patients were more likely to also receive glucocorticoids, which have been found to be associated with increased IL-10 levels, a mechanism by which they exert their anti-inflammatory effect [24].

Interestingly, despite almost $50 \%$ of critical patients receiving concomitant glucocorticoids, their IL-6 and sIL2R levels remained elevated. These results are interesting in light of preliminary reports from the RECOVERY trial, which showed a mortality benefit with the use of dexamethasone among those who received oxygen support or mechanical ventilation [25]. Our findings surrounding corticosteroids in our cohort may answer several questions recently posed in an article by Prescott and Rice [26], including whether treatment with steroids should be individualized based on clinical response or biomarkers, as well as the potential for combination therapy. First, our discovery of persistent elevation in cytokines even after glucocorticoid administration questions the overall efficacy of corticosteroids in the treatment of the COVID-19 cytokine storm. If a patient is critically ill, it is possible that corticosteroids may need to be used in combination with other therapies that target specific cytokines. Who these patients are is still difficult to conclude, but among our cohort those with high admission IL-6, IL-10, and Ddimer levels were more likely to receive glucocorticoids. Further studies confirming that utility of using these as potential biomarkers to indicate who may benefit from corticosteroids are needed.

Based on our findings, there is evidence of hyperinflammation in several immune pathways in COVID19. The persistent elevation in IL-10 in our critical patients may be a marker of ongoing immune dysregulation even after IL-6 blockade. This may signal the need for further intervention, perhaps with another immunomodulatory agent that works along a different mechanism that is activated by SARS-CoV-2 [27]. Given the high proportion of our patients with elevated sIL2R, it is possible that the dual elevation of IL-10 and sIL2R after treatment with tocilizumab may suggest the need to target $\mathrm{T}$ cell dysregulation, especially in those with unremitting severe or critical disease.

Furthermore, there were differences in IL- 6 and IL-10 trends in patients who received tocilizumab which may give us insight into the hyperinflammatory syndrome.
IL-6 seems to increase a few days after symptom onset, while IL-10 is high very early on in disease. This pattern may help us understand the origin of IL-10 production, which appears to be during the viral phase of SARS-CoV-2 infection as opposed to later on. This relatively early production of IL-10, which generally has an anti-inflammatory effect in the setting of infections, may be a reason as to why COVID-19 leads to overwhelming infection in some individuals. In vitro studies to analyze the effect of IL-10 blockade immediately after infection with SARS-CoV-2 may provide further understanding as to whether this may facilitate viral clearance.

Our cluster analyses also revealed several potential clinically relevant findings. First is that initial admission IL-6, SIL2R, and CRP values may be predictors of disease severity in COVID-19. Using this, we may be better able to predict who may need further intervention and more aggressive treatment of their cytokine storm. With the recent phase 3 COVACTA trial data regarding tocilizumab use in those with severe COVID-19, showing a trend toward decreased time to hospital discharge [28], it is possible that use of tocilizumab in combination with other therapy or in patients with certain disease markers would prove beneficial, and these are still to be discovered. Furthermore, we found that those who died had overall higher mean cytokine levels and that those with high admission IL-10, IL-6, and D-dimer levels tended to receive glucocorticoids in addition to tocilizumab. These need to be investigated as potential biomarkers for response to tocilizumab and need for adjunct therapy with glucocorticoids.

We present a large cohort of moderate, severe, and critical hospitalized COVID-19 patients with an analysis of cytokine profiles before and after immune modulation. However, our study has several limitations. First, tocilizumab was not given in the setting of a randomized controlled trial and our comparison group who did not receive it was likely a less severe group, making it an imperfect comparison. Given the urgent need for a management strategy, the treatment algorithm at this institution included consideration for tocilizumab based on oxygen requirement, which resulted in its use in many severe and critical patients. Future randomized controlled trials comparing cytokine levels in COVID-19 patients who receive tocilizumab with those who do not are needed. Additionally, the utility of other immunomodulating agents such as glucocorticoids, although not frequently utilized at this facility, may have had an impact on cytokine levels. With regard to the use of cytokine panels in the clinical management of SARS-CoV-2, future studies validating the association of cytokine levels with disease severity would be beneficial. Despite these limitations, we present the largest published cohort of serial cytokine levels in COVID-19 patients treated with tocilizumab and discuss the potential importance of IL-10 as a marker of ongoing immune dysregulation. 
Acknowledgments We would like to acknowledge all members of the COVID-19 Treatment Team, our laboratory medicine colleagues, nurses, and other frontline staff.

Authors' Contributions VA and KK acquired the data. VA, KK, and CP interpreted the data, conducted the literature review, and wrote the manuscript draft. DT and HZ performed the statistical analysis. All authors critically reviewed and approved the final manuscript.

\section{Compliance with Ethical Standards}

Conflict of Interest The authors declare that they have no conflict of interest.

\section{References}

1. Centers for Disease Control and Prevention. Coronavirus disease 2019 (COVID-19). Available from: https://www.cdc.gov/ coronavirus/2019-ncov/cases-updates/cases-in-us.html. Accessed June 26, 2020.

2. Shimabukuro-Vornhagen A, Gödel P, Subklewe M, Stemmler HJ, Schlößer HA, Schlaak M, et al. Cytokine release syndrome. J Immunother Cancer. 2018;6(1):56.

3. McGonagle D, Sharif K, O'Regan A, Bridgewood C. The role of cytokines including Interleukin-6 in COVID-19 induced pneumonia and macrophage activation syndrome-like disease. Autoimmun Rev. 2020;19:102537.

4. Mehta P, McAuley DF, Brown M, Sanchez E, Tattersall RS, Manson JJ. COVID-19: consider cytokine storm syndromes and immunosuppression. Lancet. 2020;395(10229):1033-4.

5. Short KR, Veeris R, Leijten LM, van den Brand JM, Jong VL, Stittelaar K, et al. Proinflammatory cytokine responses in extrarespiratory tissues during severe influenza. J Infect Dis. 2017;216(7):829-33.

6. Tisoncik JR, Korth MJ, Simmons CP, Farrar J, Martin TR, Katze MG. Into the eye of the cytokine storm. Microbiol Mol Biol Rev. 2012;76(1):16-32.

7. Hunter CA, Jones SA. IL-6 as a keystone cytokine in health and disease. Nat Immunol. 2015;16(5):448-57.

8. Tanaka T, Narazaki M, Kishimoto T. Interleukin (IL-6) immunotherapy. Cold Spring Harb Perspect Biol. 2018;10(8):a028456.

9. Cohen T, Nahari D, Cerem LW, Neufeld G, Levi BZ. Interleukin 6 induces the expression of vascular endothelial growth factor. J Biol Chem. 1996;271(2):736-41.

10. Herold T, Jurinovic V, Arnreich C, et al. Elevated levels of IL-6 and CRP predict the need for mechanical ventilation in COVID-19. J Allergy Clin Immunol. 146(1):128-136.e4.

11. Ruan Q, Yang K, Wang W, Jiang L, Song J. Clinical predictors of mortality due to COVID-19 based on an analysis of data of 150 patients from Wuhan, China. Intensive Care Medicine. 2020.

12. National Cancer Institute, Naples. Tocilizumab in COVID-19 pneumonia (TOCIVID-19). Available from: https://clinicaltrials.gov/ ct2/show/NCT04317092 Accessed June 16, 2020.
13. Huang C, et al. Clinical features of patients infected with 2019 Novel coronavirus in Wuhan, China. Lancet. 395(10223):497-506.

14. Couper KN, Blount DG, Riley EM. IL-10: the master regulator of immunity to infection. J Immunol. 2008;180(9):5771-7.

15. Rahmati M, Moosavi MA. Cytokine-targeted therapy in severely ill COVID-19 patients: options and cautions. EJMO. 2020;4(2):179 80.

16. Sun L, Louie MC, Vannella KM, Wilke CA, LeVine AM, Moore $\mathrm{BB}$, et al. New concepts of IL-10-induced lung fibrosis: fibrocyte recruitment and M2 activation in a CCL2/CCR2 axis. Am J Phys Lung Cell Mol Phys. 2011;300:L341-L53.

17. Monastero RN, Pentyala S. Cytokines as biomarkers and their respective clinical cutoff levels. Int J Inf Secur. 2017;2017:430948511. https://doi.org/10.1155/2017/4309485.

18. Price CC, Altice FL, Shyr Y, et al. Tocilizumab treatment for cytokine release syndrome in hospitalized COVID-19 patients: survival and clinical outcomes. CHEST

19. Lucas C, Wong P, Klein J, et al. Longitudinal analyses reveal immunological misfiring in severe COVID-19. Nature. 2020;584(7821):463-9.

20. Nishimoto N, Terao K, Mima T, Nakahara H, Takagi N, Kakehi T. Mechanisms and pathologic significances in increase in serum interleukin-6 (IL-6) and soluble IL-6 receptor after administration of an anti-IL-6 receptor antibody, tocilizumab, in patients with rheumatoid arthritis and Castleman disease. Blood. 2008;112(10): 3959-64.

21. Kleynberg RL, Schiller GJ. Secondary hemophagocytic lymphohistiocytosis in adults: an update on diagnosis and therapy. Clin Adv Hematol Oncol. 2012;10(11):726-32.

22. Luo P, Liu Y, Qiu L, Liu X, Liu D, Li J. Tocilizumab treatment in COVID-19: a single center experience [published online ahead of print, 2020 Apr 6]. J Med Virol. 2020. https://doi.org/10.1002/jmv. 25801.

23. Xu X, Han M, Li T, Sun W, Wang D, Fu B, et al. Effective treatment of severe COVID-19 patients with tocilizumab. Proc Natl Acad Sci. 2020;117(20):10970-5.

24. John M, Lim SAM, Seybold J, et al. Inhaled corticosteroids increase interleukin-10 but reduce macrophage inflammatory protein- $1 \alpha$, granulocyte-macrophage colony-stimulating factor, and interferon- $\gamma$ release from alveolar macrophages in asthma. Am J Respir Crit Care Med. 1998;157(1):256-62.

25. Dexamethasone in hospitalized patients with Covid-19 - preliminary report. New England J Med. 2020.

26. Prescott HC, Rice TW. Corticosteroids in COVID-19 ARDS: evidence and hope during the pandemic. JAMA. 2020;324:1292-5.

27. Ingraham NE, Lotfi-Emran S, Thielen BK, et al. Immunomodulation in COVID-19. Lancet Respir Med.

28. Roche provides an update on the phase III COVACTA trial of Actemra/RoActemra in hospitalised patients with severe COVID19 associated pneumonia. https://www.roche.com/investors/ updates/inv-update-2020-07-29.htm. Date accessed: September $22,2020$.

Publisher's Note Springer Nature remains neutral with regard to jurisdictional claims in published maps and institutional affiliations. 